\title{
A Minimized Synthetic Carbon Fixation Cycle
}

\section{Supporting Information}

Lu Xiao ${ }^{1,2}$, Guoxia Liu ${ }^{1}$, Fuyu Gong ${ }^{1,2}$, Huawei Zhu ${ }^{1,2}$, Zhen Cai ${ }^{1}$, Yin $\mathrm{Li}^{{ }^{*}}$

${ }^{1}$ CAS Key Laboratory of Microbial Physiological and Metabolic Engineering, State Key Laboratory of Microbial Resources, Institute of Microbiology, Chinese Academy of Sciences, Beijing 100101, China

${ }^{2}$ University of Chinese Academy of Sciences, Beijing 100049, China

*Corresponding author. E-mail: yli@im.ac.cn 


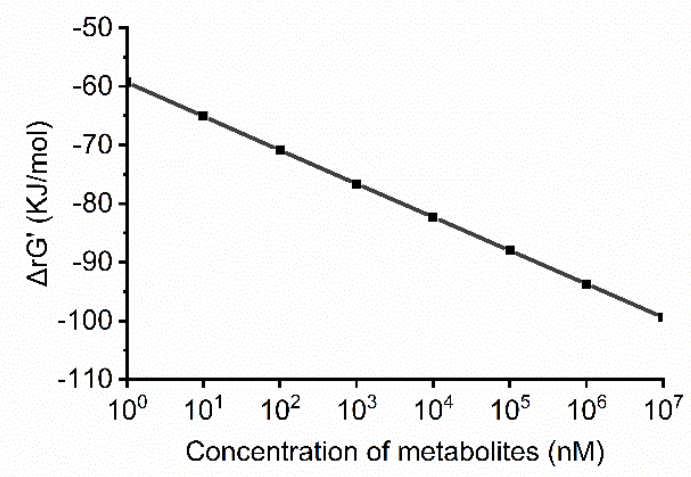

Fig. S1. Thermodynamic analysis of the POAP cycle with the operating intracellular metabolites concentrations. The Gibbs free energies of reactions were calculated using an ionic strength $I=$ 0.25 at $\mathrm{pH} 7.0$, and assuming the follow metabolite concentration: $\mathrm{ATP}=1.2 \mathrm{mM} ; \mathrm{ADP}=0.2 \mathrm{mM}$; AMP $=0.2 \mathrm{mM} ;$ CoASH $=0.5 \mathrm{mM} ;$ orthophosphate $=0.01 \mathrm{mM}$; diphosphate $=0.01 \mathrm{mM}$. The concentrations of other metabolites involved in the POAP cycle are assumed in the range of $1 \mathrm{nM}$ to $10 \mathrm{mM}$ according to literature \{reference\}. These values were calculated using eQuilibrator (https://equilibrator.weizmann.ac.il/). The $\Delta \mathrm{rG}^{\prime} \mathrm{m}$ are the addition of all reactions.

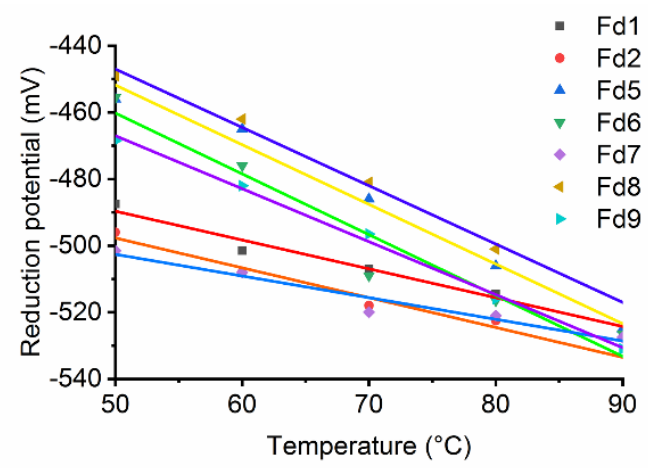

Fig. S2. The reduction potential of Fds from different species tested in this work at different temperatures. 
A

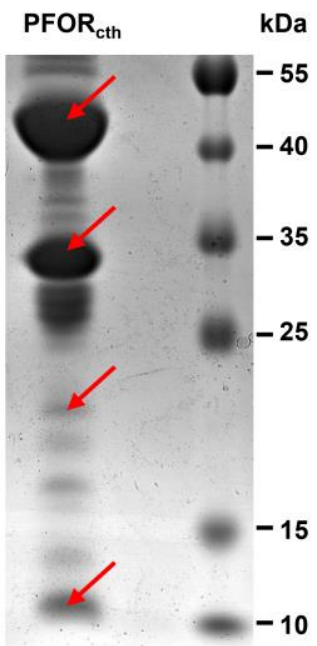

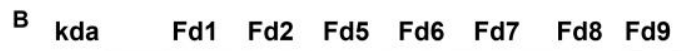

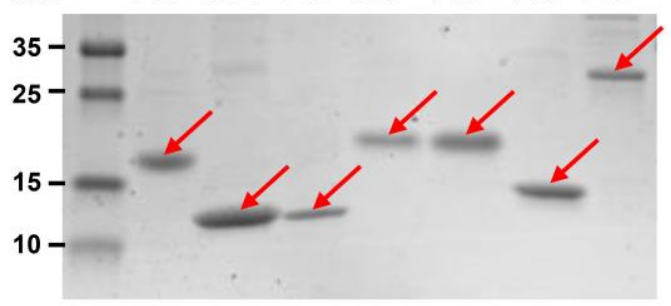

Fig. S3. SDS-PAGE of PFOR ${ }_{c t h}(A)$ and Fds purified in this work (B). The molecular weights of Fd1, $\mathrm{Fd} 2, \mathrm{Fd} 5, \mathrm{Fd} 6$ and Fd7 are less than $10 \mathrm{kDa}$, but the corresponding bands migrated above the 10 kDa marker band. This phenomenon is commonly observed due to the high negative charge of Fds.

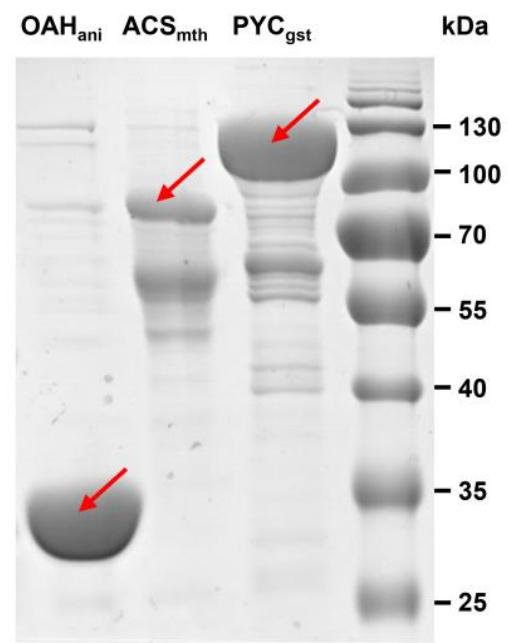

Fig. S4. SDS-PAGE of OAH ani, $A C S_{m t h}$ and PYC $g$ st. 
A

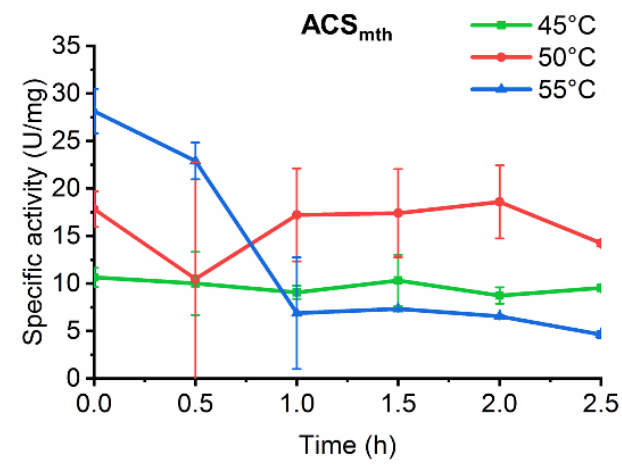

C

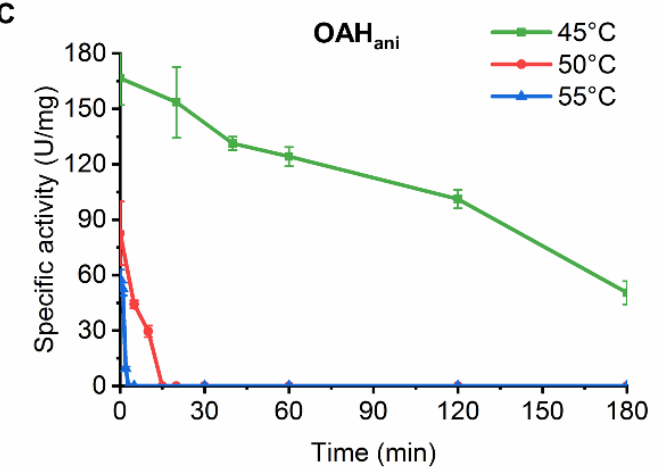

B

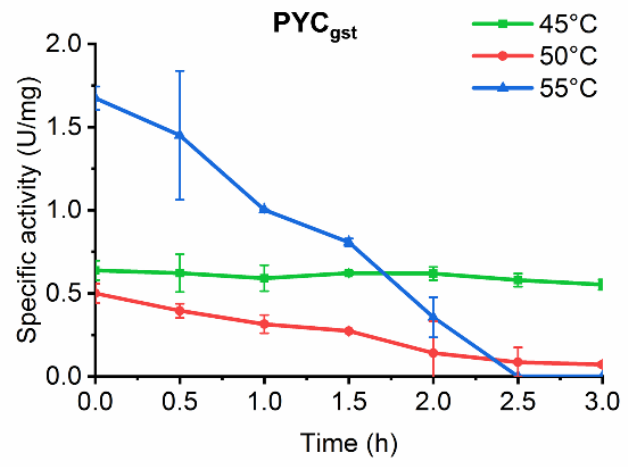

Fig. S5. Temperature stability of $A C S_{m t h}(A), P_{g} C_{g s t}(B)$ and $O A H_{a n i}(C)$. 


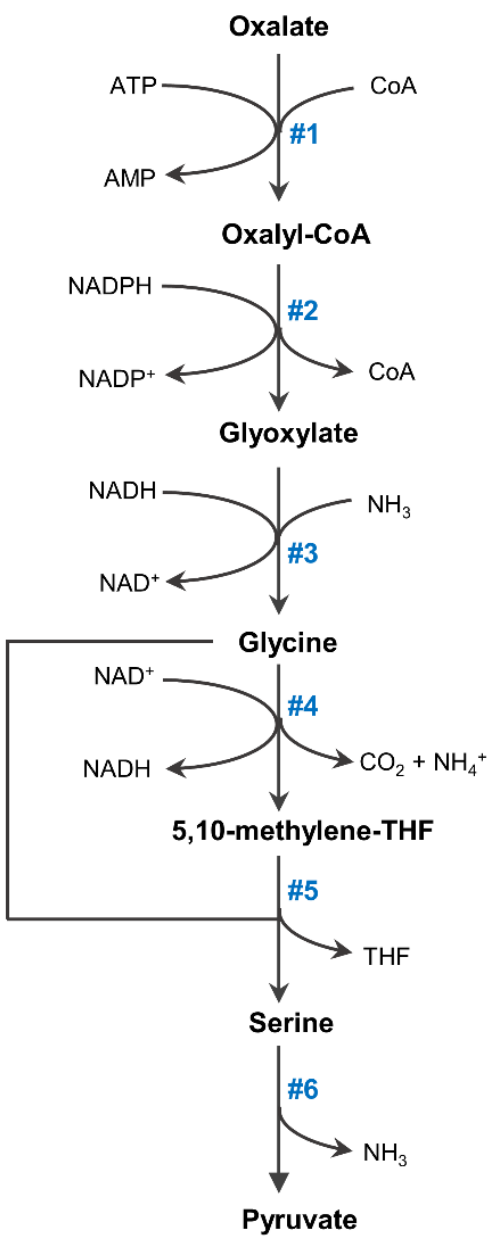

Fig. S6. The pathway for converting oxalate into pyruvate. \#1 oxalate-CoA ligase; \#2 glyoxylate dehydrogenase; \#3 glycine dehydrogenase; \#4 glycine cleavage system; \#5 glycine hydroxymethyltransferase; \#6 L-serine ammonia lyase 
A

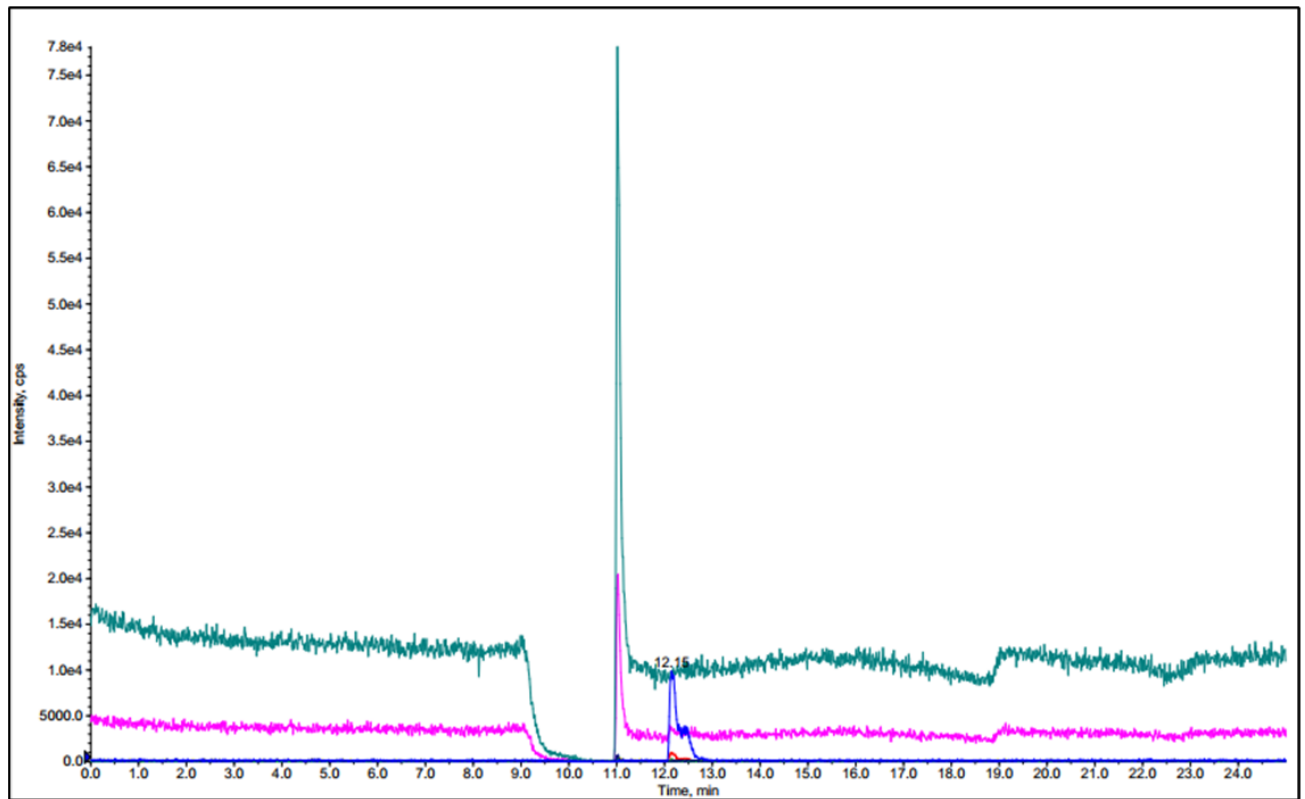

B

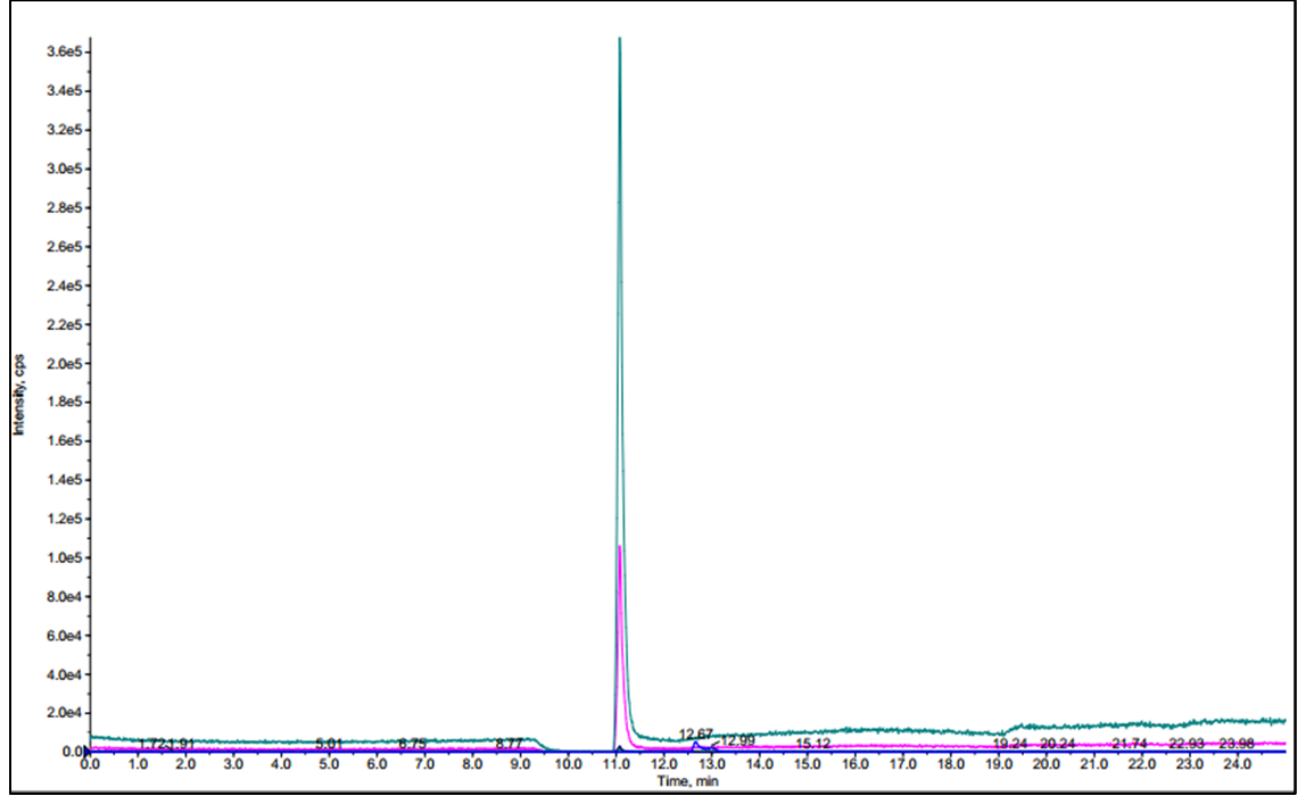

Fig. S7. The LC-MS spectrum of oxalate produced by the POAP cycle (A) and the oxalate standard (B). 


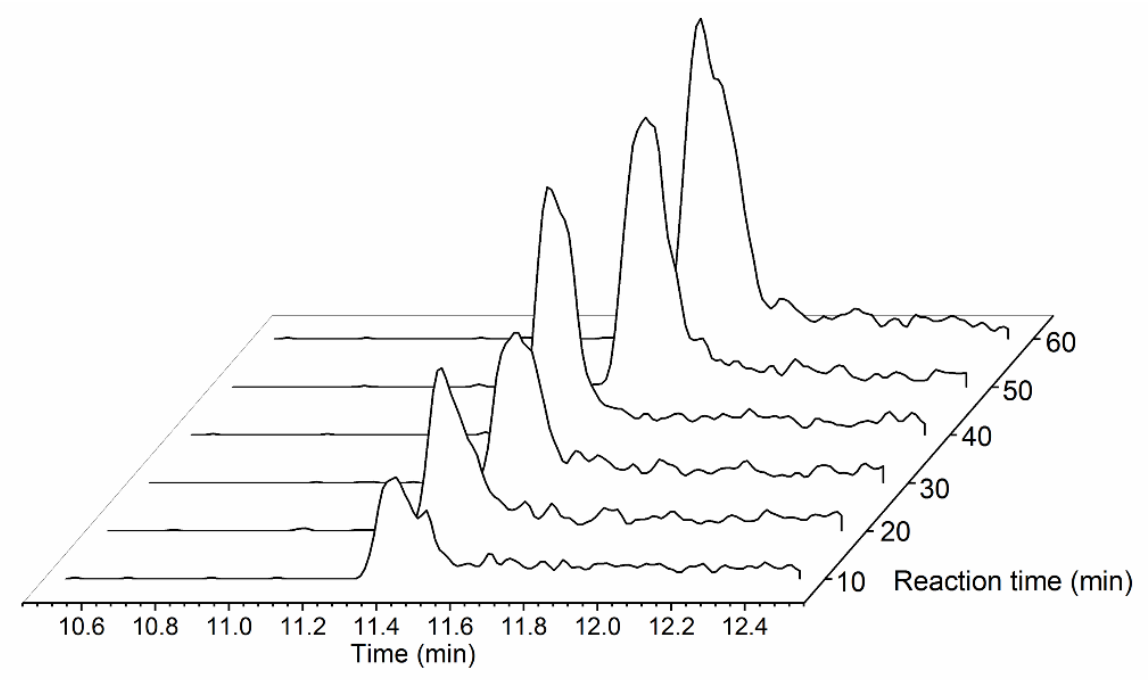

Fig. S8. Peaks of ${ }^{13} \mathrm{C}$-labeled oxalate produced by the POAP cycle at different reaction times. As the reaction time increases, the peak area of ${ }^{13} \mathrm{C}$-labeled oxalate increases. 
Table S1. Sources and redox potentials $\left(E^{0^{\prime}}\right)$ of electron donors used to support the reductive carboxylation of pyruvate synthase in the present study. $\mathrm{Fd}^{*}$ indicated that the corresponding $\mathrm{Fd}$ could not be successfully expressed.

\begin{tabular}{|c|c|c|c|c|}
\hline & Potential (mV) & source & & Reference \\
\hline Fd1 & -488 & Hydrogenobacter thermophiles & natural & this work \\
\hline $\mathrm{Fd} 2$ & -496 & Hydrogenobacter thermophiles & natural & this work \\
\hline $\mathrm{Fd} 3 *$ & - & Chlorobium tepidum & natural & this work \\
\hline $\mathrm{Fd} 4 *$ & - & Chlorobium tepidum & natural & this work \\
\hline $\mathrm{Fd} 5$ & -456 & Pyrodictium delaneyi & natural & this work \\
\hline $\mathrm{Fd} 6$ & -456 & Aquifex aeolicus & natural & this work \\
\hline Fd7 & -502 & Aquifex aeolicus & natural & this work \\
\hline Fd8 & -449 & Clostridium thermocellum & natural & this work \\
\hline Fd9 & -469 & Clostridium thermocellum & natural & this work \\
\hline MV & -446 & - & artificial & 33 \\
\hline NADPH & $-300^{\sim}-400$ & - & natural & 34 \\
\hline
\end{tabular}


Table. S2. List of OAH, ACS and PYC enzymes that were expressed, purified and tested in this work. PYC-gse, OAH-ang and ACS-mth were employed in the POAP cycle.

\begin{tabular}{|c|c|c|c|}
\hline Enzyme & Species & $\begin{array}{l}\text { Tested } \\
\text { temperature } \\
\left({ }^{\circ} \mathrm{C}\right)\end{array}$ & $\begin{array}{l}\text { Specific } \\
\text { activity (U/mg) }\end{array}$ \\
\hline PYC-gst & Geobacillus stearothermophilus & 50 & $0.500 \pm 0.05$ \\
\hline PYC-mja & Methanocaldococcus jannaschii & 80 & $3.341 \pm 0.137$ \\
\hline PYC-mth & $\begin{array}{l}\text { Methanothermobacter } \\
\text { thermautotrophicus }\end{array}$ & 37 & $1.447 \pm 0.302$ \\
\hline OAH-bfu & Botrytis cinerea & 25 & $1.853 \pm 0.052$ \\
\hline OAH-ang & Aspergillus niger & 50 & $82.603 \pm 17.189$ \\
\hline OAH-tat & Talaromyces atroroseus & 40 & $0.313 \pm 0.016$ \\
\hline OAH-tce & Talaromyces cellulolyticus & 40 & 0 \\
\hline ACS-mth-0554 & Methanosaeta thermophila & 55 & $0.087 \pm 0.029$ \\
\hline ACS-mth & Methanosaeta thermophila & 50 & $17.830 \pm 1.869$ \\
\hline ACS-mth-1413 & Methanosaeta thermophila & 55 & $0.024 \pm 0.014$ \\
\hline ACS-mth-1194 & Methanosaeta thermophila & 60 & $1.034 \pm 0.002$ \\
\hline ACS- methe -1 & $\begin{array}{l}\text { Methanothermobacter } \\
\text { thermautotrophicus }\end{array}$ & 55 & $0.014 \pm 0.004$ \\
\hline ACS- methe -2 & $\begin{array}{l}\text { Methanothermobacter } \\
\text { thermautotrophicus }\end{array}$ & 55 & $0.304 \pm 0.024$ \\
\hline ACS-afu & Archaeoglobus fulgidus & 80 & $1.308 \pm 0.053$ \\
\hline ACS-mta & Moorella thermoacetica & 60 & $0.053 \pm 0.004$ \\
\hline ACS-bja-1 & Bradyrhizobium japonicum & 26 & $1.281 \pm 0.012$ \\
\hline ACS-bja-2 & Bradyrhizobium japonicum & 26 & $0.269 \pm 0.138$ \\
\hline
\end{tabular}




\begin{tabular}{llcl}
\hline ACS-mse & Metallosphaera sedula & 65 & $0.230 \pm 0.037$ \\
ACS-mro & Melioribacter roseus & 47 & $0.011 \pm 0.003$ \\
ACS-pai & Pyrobaculum aerophilum & 80 & $0.736 \pm 0.004$ \\
ACS-bsu & Bacillus subtilis & 37 & $0.005 \pm 0.006$ \\
\hline
\end{tabular}

Table S3. Number of amino acid residues and molecular weight of Fds used in this work.

\begin{tabular}{ccc}
\hline Ferredoxin & $\begin{array}{c}\text { Amino acid } \\
\text { residues }\end{array}$ & Molecular weight (kDa) \\
\hline Fd1 & 72 & 7.9 \\
Fd2 & 67 & 7.5 \\
Fd5 & 81 & 9.0 \\
Fd6 & 76 & 8.4 \\
Fd7 & 80 & 9.1 \\
Fd8 & 122 & 13.4 \\
Fd9 & 226 & 24.9 \\
\hline
\end{tabular}




\section{Supplementary Text}

Supplementary Text 1: Calculation of the total turnover numbers of the enzymes involved in the POAP cycle.

In this study, the volume of the POAP cycle reaction system was $300 \mu \mathrm{L}$. The amount of enzymes added to the POAP cycle reaction system were $65.300 \mu \mathrm{g}$ of PFOR $\mathrm{R}_{\mathrm{cth}}, 46.560 \mu \mathrm{g}$ of $\mathrm{PYC}_{\mathrm{gst}}, 12.745$

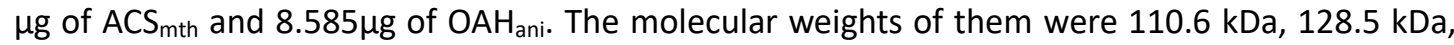
$75.4 \mathrm{kDa}$ and $32.4 \mathrm{kDa}$, respectively. Therefore, the total amount of the four enzymes was calculated to be $1.378 \mathrm{nmol}$. Since $6.9 \mathrm{nmol}$ oxalate $(23 \mu \mathrm{M})$ was produced after $20 \mathrm{~min}$, the total numbers of the enzymes involved in the POAP cycle was 5 ( $m o l$ of oxalate obtained divided by mol of the four enzymes).

Supplementary Text 2: DNA sequences of codon-optimized pfor $\left(\mathrm{PFOR}_{\mathrm{cth}}\right)$, oah $\left(\mathrm{OAH}_{\mathrm{ani}}\right)$, acs $\left(\mathrm{ACS}_{\mathrm{mth}}\right)$, pyc $\left(\mathrm{PYC}_{\mathrm{gst}}\right), f n r\left(\mathrm{FNR}_{\mathrm{cte}}\right), l d h\left(\mathrm{LDH}_{\mathrm{bco}}\right)$ and $f d(\mathrm{Fd})$ genes in this work.

1. pfor

pfor alpha subunit

ATGGGTATCCGTGAACGTCTGTCTGGTAACGAAGCTACCGCTATCGCTATGCGTCAGATCAACCCGGACGTT GTTGCTGCTTTCCCGATCACCCCGTCTACCGAAATCCCGCAGTACTTCTCTTCTTACGTTGCTGACGGTCTGG TTGACACCGAATTCGTTGCTGTTGAATCTGAACACTCTGCTATGTCTGCTTGCATCGGTGCTCAGGCTGCTGG TGCTCGTGCTATGACCGCTACCTCTGCTAACGGTCTGGCTTACATGTGGGAAGCTCTGTACATCGCTGCTTCT ATGCGTCTGCCGATCGTTCTGGCTGCTGTTAACCGTGCTCTGTCTGGTCCGATCAACATCCACAACGACCACT CTGACACCATGGGTGCTCGTGACTCTGGTTGGATCCAGCTGTACTCTGAAAACAACCAGGAAGCTTACGAC AACATGCTGATGGCTCACCGTATCGGTGAACACCCGGACGTTATGCTGCCGGTTATGGTTTGCCAGGACGGT TTCATCACCTCTCACGCTATCGAAAACATCGAACTGGTTGAAGACGAAAAAGTTAAAGCTTTCGTTGGTGAA TACAAACCGACCCACTACCTGCTGGACCGTGAAAACCCGATCTCTGTTGGTCCGCTGGACCTGCAGATGCAC TACTTCGAACACAAACGTCAGCAGGCTCAGGCTATGGAAAACGCTAAAAAAGTTATCCTGGAAGTTGCTGA 
AGAATTCTACAAACTGACCGGTCGTAAATACGGTTTCTTCGAAGAATACAAAACCGACGACGCTGACGTTGC TATCGTTGTTATGAACTCTACCGCTGGTACCGTTAAATACGTTATCGACGAATACCGTGTTAAAGGTAAAAAA GTTGGTCTGATCAAACCGCGTGTTTTCCGTCCGTTCCCGGTTGACGAACTGGCTCAGGCTCTGTCTAAATTC AAAGCTGTTGCTGTTATGGACAAAGCTGACTCTTTCAACGCTGCTGGTGGTCCGCTGTTCACCGAAGTTACC TCTGCTCTGTTCACCAAAGGTGTTTTCGGTCCGAAAGTTATCAACTACAAATTCGGTCTGGGTGGTCGTGAC GTTAAAGTTGACGACATCGAAGTTGTTTGCGAAAAACTGCTGGAAATCGCTTCTACCGGTAAAGTTGACTCT GTTTACAACTACCTGGGTGTTCGTGAATAA

pfor beta subunit ATGGCTTACAACCTGAAAGAAGTTGCTAAAAAACCGGAACGTCTGACCGGTGGTCACCGTATGTGCGCTGG TTGCGGTGCTCCGATCGTTGTTCGTCAGGTTCTGAAAGCTCTGAAACCGGAAGACCACGCTGTTATCTCTGC TGCTACCGGTTGCCTGGAAGTTTCTACCTTCATCTACCCGTACACCGCTTGGAAAGACTCTTTCATCCACTCT GCTTTCGAAAACACCGGTGCTACCATCTCTGGTGCTGAAGCTGCTTACAAAGTTCTGAAAAAAAAAGGTAA AATCGAAGGTGAAACCAAATTCATCGCTTTCGGTGGTGACGGTGGTACCTACGACATCGGTCTGCAGGCTCT GTCTGGTGCTATGGAACGTGGTCACGACATGGTTTACGTTTGCTACGACAACGGTGCTTACATGAACACCGG TATCCAGCGTTCTTCTGCTACCCCGAAATACGCTGACACCACCACCTCTCCGGTTGGTAAAAAAATCCCGGGT AAAATGCAGCCGCGTAAAGACCTGACCGAAGTTCTGGTTAACCACCGTATCCCGTACGTTGCTCAGACCGCT CCGTTCGGTAACATGAAAGACCTGTACGAAAAAGCTGAAAAAGCTATCTACACCCCGGGTCCGGCTTTCCTG AACGTTCTGGCTCCGTGCCCGCGTGGTTGGCGTTACAACACCCCGGACCTGATGGAACTGTCTAAACTGGC TGTTGAAACCTGCTTCTGGCCGCTGTACGAAGTTATCGACGGTAAATACATCATCAACTACAAACCGAAAGA AAAAGTTCCGGTTAAAGAATTCCTGAAACTGCAGGGTCGTTTCAAACACCTGTTCAAAGCTGGTAACGAATA CATGCTGGAAGAAATCCAGAAAGAAGTTGACCTGCGTTGGGAACGTCTGCTGAAACTGGCTGGTGAAGCT TAA

pfor gamma subunit ATGGGTAAAGTTGTTGAAATCCGTTGGCACGGTCGTGGTGGTCAGGGTGCTAAAACCGCTTCTCTGCTGCT GGCTGACGCTGCTTTCAACACCGGTAAATACATCCAGGGTTTCCCGGAATACGGTCCGGAACGTATGGGTGC 
TCCGATCACCGCTTACAACCGTATCTCTGACGAAAAACTGACCATCCACTCTAACATCTACGAACCGGACTAC GTTGTTGTTGTTGACGACACCCTGCTGACCTCTGTTGACGTTACCGCTGGTCTGAAAGAAGACGGTGCTATC ATCGTTAACACCCCGAAAACCCCGGACGAAATCCGTCCGCTGCTGAAAGGTTACAAAGGTAAAGTTTGCAC CATCGACGCTCGTAAAATCTCTATCGAAACCCTGGGTAAATACTTCCCGAACACCCCGATGCTGGGTGCTGTT GTTAAAGTTTCTAAAATCATGGACGAAGAAGAATTCCTGAAAGACATGGTTGAATCTTTCAAACACAAATTC GCTAACAAACCGGAAGTTGTTGAAGGTAACATCAAAGCTCTGGAACGTTCTATGCAGGAAGTTAAAGGTCT GTAA

pfor delta subunit

ATGTCTAAAGAACTGCGTGACGTTAAACCGGACGTTACCTGGAAAGAAATCACCTCTGGTGGTGTTATCGAC TCTCCGGGTAACGCTCACCTGTTCAAAACCGGTGACTGGCGTTCTATGAAACCGGTTTGGAACGAAGAAAA ATGCAAACAGTGCCTGCTGTGCAACCCGGTTTGCCCGGACTCTTCTATCATGGTTTCTGAAGAAGGTAAAAT GACCGGTATCGACTACGACCACTGCAAAGGTTGCGGTATCTGCTCTAAAGTTTGCCCGTTCAAAGCTATCGA CTTCGTTGAAGAAGTTTAA

2. oah

ATGGTTACCGGTGCTTCTAAACTGCGTAAACAGCTGCGTGAAACCAACGAACTGCTCGTTTGCCCAGGTGTG TACGACGGCCTGTCTGCTCGTATCGCTATCAACCTGGGTTTCAAAGGTATGTACATGACCGGTGCTGGTACCA CCGCTTCTCGTCTGGGTATGGCTGACCTGGGTCTGGCTCACATCTACGACATGAAAACCAACGCTGAAATGA TCGCTAACCTGGACCCGTACGGTCCGCCGCTGATCGCTGACATGGACACCGGTTACGGTGGTCCGCTGATG GTTGCTCGTTCTGTTCAGCAGTACATCCAGGCTGGTGTTGCTGGTTTCCACATCGAAGACCAGATCCAGAAC AAACGTTGCGGTCACCTGGCTGGTAAACGTGTTGTTACCATGGACGAATACCTGACCCGTATCCGTGCTGCT AAACTGACCAAAGACCGTCTGCGTTCTGACATCGTTCTGATCGCTCGTACCGACGCTCTGCAGCAGCACGGT TACGACGAATGCATCCGTCGTCTGAAAGCTGCTCGTGACCTGGGTGCTGACGTTGGTCTGCTGGAAGGTTT CACCTCTAAAGAAATGGCTCGTCGTTGCGTTCAGGACCTGGCTCCGTGGCCGCTGCTGCTGAACATGGTTG AAAACGGTGCTGGTCCGGTTATCTCTGTTGACGAAGCTCGTGAAATGGGTTTCCGTATAATGATCTTCAGCTT CGCTTGCATCACTCCGGCTTACATGGGTATCACCGCTGCTCTGGAACGTCTGAAAAAAGACGGTGTTGTTGG 
TCTGCCGGAAGGTATGGGTCCGAAAAAACTCTTTGAAGTCTGCGGCCTGATGGACTCTGTTCGTGTTGACA CCGAAGCTGGTGGTGACGGTTTCGCTAACGGTGTTTAA

\section{3. acs}

ATGACCGTTGAAGAATGCCACAAAATGGCTGAAGAAAAAGCTGCTACCACCGCTGTTCTGCTGGAAGAAAC CCGTGTTTTCCACCCGCCGAAAGAACTGGCTGAAAACTCTAACGTTATGCAGTGGATGAAAAAAAAAGGTT TCACCTCTGAACGTGAAATGCGTGCTTGGACCGGTCAGCACTACATCGAATTCTGGGACGAAATGGCTAAAA CCTACGCTGACTGGTTCGAACCGTACGCTCAGATCCTGGAATGGAAACCGCCGTACGCTCGTTGGTTCGTTG GTGGTAAATGCAACGTTGCTTACAACGCTGTTGACCGTCACGCTAAAGGTGCTAAAAAAGACAAAGTTGCT TACATCTTCGTTCCGGAACCGACCGACCAGCAGGTTCGTAAAATCACCTACCTGGACCTGTACAAAGCTGTT AACAAATTCGCTAACGGTCTGAAATCTCTGGGTGTTAAAAAAGGTGACCGTGTTTCTATCTACATGCCGATGA TCCCGGAAACCCCGATCGCTATGCTGGCTTGCGCTAAAATCGGTGCTATCCACTCTGTTGTTTTCTCTGGTTTC TCTGCTGGTGGTCTGCAGTCTCGTGTTCTGGACGCTGAAGCTAAAGTTGTTGTTACCACCGACGGTTTCTAC CGTCGTGGTAAACCGCTGCCGCTGAAACCGAACGTTGACGAAGCTGTTCAGAACGCTCCGTCTGTTGAAAA AGTTGTTGTTGTTAAACGTGCTGGTCTGGACGTTCCGATGAAAGAAGGTCGTGACATCTGGTACCACGAACT GGTTGCTAACCAGCCGGACGAATGCGAAACCGAAAAAATGGACTCTGAAGACCGTCTGTTCATCCTGTACA CCTCTGGTACCACCGGTAAACCGAAAGGTATCGAACACGTTCACGGTGGTTACTGCGTTACCCCGGCTCAGA CCACCCACTGGGTTTTCGACCTGAAAGACGACGACGTTTGGTGGTGCACCGCTGACGTTGGTTGGATCACC GGTCACTCTTACGTTGTTTACGGTCCGCTGTGCCTGGGTGCTACCTCTATCCTGTACGAAGGTGCTCCGGACT ACCCGGACTTCGGTCGTTGGTTCTCTATCATCCAAGAACACAAAGTTTCTGTCTTCTACACCGCGCCGACCGC TATCCGTATGTTCATGAAAGCTGGTGAACAGTGGCCGCAGAAATACGACCTGTCTTCTCTGCGTCTGCTGGG TTCTGTTGGTGAACCGATCAACCCGGAAGCTTGGATCTGGTACCGTAAATACTTCGGTTCTGACCGTTGCCC GATCATGGACACCTGGTGGCAGACCGAAACCGGTTGCTTCGTTGTTTCTCCGCTGCCGATCACCCCGCTGAA ACCGGGTTCTCCGACCTTCCCGCTCCCAGGCTTCAACACCGACATATACGACGAAGACGCTAACCCGGTTCC GCCGGGTCAGGGTGGTAACATCGTTAACCCGACCCCGTGGCCGTCTATGCTGCGTGCTTTCTACAAAGACCC GGAACGTTACATGAAAGAATACTGGCAGATGTACTGGGACATCCGTCCAGGTGTATACCTGGCAGGTGACA AAGCTACCCGTGACAAAGACGGTTACTTCTTCATCCAGGGTCGTATCGACGACGTTCTGAAAGTTGCTGGTC 
ACCGTATCTCTAACGCTGAAGTTGAATCTGCTCTGGTTTCTCACCCGGCTGTTGCTGAAGCTGCTGTTATCGG TAAACCGGACGAAGTTAAAGGTGAAGTTATCGTTGCTTTCGTTATCCTGCGTGAAGGTGTTAAAGAATCTGA AGACCTGAAAAAAGAACTGGCTAAACACGTTCGTTCTGTTCTGGGTCCGGTTGCTTACCCGGAAATCGTTTA CTTCGTTAAAGACGTTCCGAAAACCCGTTCTGGTAAAATCATGCGTCGTGTTATCAAAGCTAAAGCTCTGGG TAACCCGGTTGGTGACATCTCTGCTCTGGCTAACCCGGAAGCTGTTGACGCTATCCCGCTGATCAAATAA

\section{4. pyc}

ATGCGTACCCGTCGTATCCGTAAAGTTCTGGCTGCTAACCGTGGTGAAATCGCTATCCGTGTTTTCCGTGCTT GCACCGAACTGGACATCCGTACCGTTGCTATCTACTCTAAAGAAGACGCTGGTTCTTACCACCGTTACAAAG CTGACGAAGCTTACCTGGTTGGTGAAGGTAAAAAACCGATCGAAGCTTACCTGGACATCGAAGGTATCATC GAAATCGCTAAAGCTCACGACGTTGACGCTATCCACCCGGGTTACGGTTTCCTGTCTGAAAACATCCAGTTC GCTAAACGTTGCCGTGAAGAAGGTATCATCTTCATCGGTCCGAACGAAGAACACCTGGACATGTTCGGTGA CAAAGTTAAAGCTCGTCACGCTGCTATGAAAGCTGGTATCCCGGTTATCCCGGGTTCTGACGGTCCGGTTGG TGGTCTGGAAGACGTTGTTCGTTTCGCTGAAACCCACGGTTACCCGATCATCATCAAAGCTGCTCTGGGTGG TGGTGGTCGTGGTATGCGTATCGTTCGTTCTAAATCTGAAGTTAAAGAAGCTTTCGAACGTGCTAAATCTGAA GCTAAAGCTGCTTTCGGTTCTGACGACGTTTACGTTGAAAAACTGATCGAAAAACCGAAACACATCGAAGT TCAGATCCTGGGTGACCACGAAGGTAACATCGTTCACCTGTACGAACGTGACTGCTCTGTTCAGCGTCGTCA CCAGAAAGTTGTTGAAGTTGCTCCGTCTGTTTCTCTGTCTGACGAACTGCGTGAACGTATCTGCGAAGCTGC TGTTCGTCTGATGCGTTCTGTTGGTTACGTTAACGCTGGTACCGTTGAATTCCTGGTTTCTGGTGACGAATTC TACTTCATCGAAGTTAACCCGCGTATCCAGGTTGAACACACCATCACCGAAATGATCACCGGTATCGACATCG TTCAGTCTCAGATCCTGATCGCTGACGGTTTCTCTCTGCACTCTCCGGAAGTTGGTATCCCGAAACAGGAAG ACATCCGTATCAACGGTTACGCTATCCAGTCTCGTGTTACCACCGAAGACCCGCTGAACAACTTCATGCCGGA CACCGGTAAAATCATGGCTTACCGTTCTGGTGGTGGTTTCGGTGTTCGTCTGGACGCTGGTAACGGTTTCCA GGGTGCTGTTATCACCCCGTACTACGACTCTCTGCTGGTTAAAGTTTCTACCTGGGCTCTGACCTTCGAACAG GCTGCTCGTAAAATGCTGCGTAACCTGCGTGAATTCCGTATCCGTGGTATCAAAACCAACATCCCGTTCCTGG AAAACGTTGTTCAGCACCCGAAATTCCTGTCTGGTGAATACGACACCTCTTTCATCGACACCACCCCGGAAC TGTTCGTTTTCCCGCGTCGTAAAGACCGTGGTACCAAAATGCTGACCTACATCGGTACCGTTACCGTTAACGG 
TTTCCCGGGTATCGGTAAAAAAAAAAAACCGGTTTTCGACAAACCGCGTGTTCCGAAAGTTTCTCAGACCG AACCGATCCCGGCTGGTACCAAACAGATCCTGGACGAACGTGGTCCGGAAGGTCTGGTTCGTTGGATCCAG GAACAGCCGCGTGTTCTGCTGACCGACACCACCTTCCGTGACGCTCACCAGTCTCTGCTGGCTACCCGTGTT CGTACCATCGACCTGCTGCGTATCGCTGAACCGACCGCTCGTCTGCTGCCGAACCTGTTCTCTCTGGAAATGT GGGGTGGTGCTACCTTCGACGTTGCTTACCGTTTCCTGAAAGAAGACCCGTGGGACCGTCTGCTGAAACTG CGTGAACGTATCCCGAACGTTCTGTTCCAGATGCTGCTGCGTTCTGCTAACGCTGTTGGTTACAAAAACTACC CGGACAACGTTATCCGTGAATTCGTTGACAAATCTGCTCAGGCTGGTATCGACGTTTTCCGTATCTTCGACTC TCTGAACTGGGTTAAAGGTATGACCGTTGCTATCGACGCTGTTCGTCAGTCTGGTAAAATCGCTGAAGCTGC TATCTGCTACACCGGTGACATCCTGGACCCGAACCGTCCGAAATACAACCTGGACTACTACAAAGCTCTGGC TAAAGAACTGGAACAGGCTGGTGCTCACATCCTGGGTATCAAAGACATGGCTGGTCTGCTGAAACCGCAGG CTGCTTACGTTCTGATCTCTGCTCTGAAAGAAACCGTTTCTATCCCGATCCACCTGCACACCCACGACACCTCT GGTAACGGTATCTACACCTACGCTAAAGCTATCGAAGCTGGTGTTGACATCGTTGACGTTGCTGTTTCTTCTAT GGCTGGTCTGACCTCTCAGCCGTCTGCTAACACCCTGTACTACGCTCTGGAAGGTACCGAACGTGCTCCGGA AGTTGACATCTACGGTCTGGAACAGCTGGCTCGTTACTGGGAAGACGTTCGTAAATTCTACCAGGAATTCGA ATCTGGTATGAACGCTCCGCACACCGAAGTTTACATGCACGAAATGCCGGGTGGTCAGTACTCTAACCTGCA GCAGCAGGCTAAAGCTGTTGGTCTGGGTGACCGTTGGGACGAAGTTAAAGAAATGTACCGTCGTGTTAACG ACCTGTTCGGTGACATCGTTAAAGTTACCCCGTCTTCTAAAGTTGTTGGTGACATGGCTCTGTACATGGTTCA GAACAACCTGACCGAACAGGACATCTTCGAACGTGGTGAAACCCTGAACTTCCCGGACTCTGTTGTTGAAT TCTTCGAAGGTTACCTGGGTCAGCCGCACGGTGGTTTCCCGAAAGAACTGCAGCGTATCATCCTGAAAGGT CGTGAACCGATCACCGTTCGTCCGGGTGAACTGCTGGAACCGGTTGACTTCGAACAGATCAAACGTGAACT GTACGACAAACTGGGTCGTGAAGTTACCGACTTCGACGCTATCGCTTACGCTCTGTACCCGAAAGTTTTCCT GGAATACGCTGAAACCGTTGAAAAATACGGTGACATCTCTGTTCTGGACACCCCGACCTTCCTGTACGGTAT GCGTCTGGGTGAAGAAATCGAAGTTGAAATCGAACGTGGTAAAACCCTGATCGTTAAACTGGTTTCTATCGG TCAGCCGCAGGCTGACGGTACCCGTGTTGTTTACTTCGAACTGAACGGTCAGCCGCGTGAAGTTATCATCCG TGACGAATCTATCAAAGCTGCTGTTGCTGAACGTATCAAAGCTGACCGTACCAACCCGAACCACATCGCTGC TACCATGCCGGGTACCGTTGTTAAAGTTCTGGTTGAAAAAGGTGAAAAAGTTGACAAAGGTGACCACCTGA TGGTTACCGAAGCTATGAAAATGGAAACCACCGTTCAGGCTCCGTTCGCTGGTATCGTTAAAGACATCTACG 
TTAAATCTGGTGACGCTATCCAGGCTGGTGACCTGCTGATCGAACTGTCTAAATAA

\section{5. fnr}

ATGCTGGACATCCACAACCCGGCTACCGACCACCACGACATGCGTGACCTGACCATCATCGGTGGTGGTCCG ACCGGTATCTTCGCTGCTTTCCAGTGCGGTATGAACAACATCTCTTGCCGTATCATCGAATCTATGCCGCAGCT GGGTGGTCAGCTGGCTGCTCTGTACCCGGAAAAACACATCTACGACGTTGCTGGTTTCCCGGAAGTTCCGG CTATCGACCTGGTTGAATCTCTGTGGGCTCAGGCTGAACGTTACAACCCGGACGTTGTTCTGAACGAAACCG TTACCAAATACACCAAACTGGACGACGGTACCTTCGAAACCCGTACCAACACCGGTAACGTTTACCGTTCTC GTGCTGTTCTGATCGCTGCTGGTCTGGGTGCTTTCGAACCGCGTAAACTGCCGCAGCTGGGTAACATCGACC ACCTGACCGGTTCTTCTGTTTACTACGCTGTTAAATCTGTTGAAGACTTCAAAGGTAAACGTGTTGTTATCGT TGGTGGTGGTGACTCTGCTCTGGACTGGACCGTTGGTCTGATCAAAAACGCTGCTTCTGTTACCCTGGTTCA CCGTGGTCACGAATTCCAGGGTCACGGTAAAACCGCTCACGAAGTTGAACGTGCTCGTGCTAACGGTACTAT AGACGTGTACCTAGAAACCGAAGTTGCTTCTATCGAAGAATCTAACGGTGTTCTGACCCGTGTTCACCTGCG TTCTTCTGACGGTTCTAAATGGACCGTTGAAGCTGACCGTCTGCTGATCCTCATAGGCTTCAAATCTAACCTC GGTCCGCTGGCTCGTTGGGACCTGGAACTGTACGAAAACGCTCTGGTTGTTGACTCTCACATGAAAACCTCT GTTGACGGTCTGTACGCTGCTGGTGACATCGCTTACTACCCGGGTAAACTGAAAATCATCCAGACCGGTCTG TCTGAAGCTACCATGGCTGTTCGTCACTCTCTGTCTTACATCAAACCGGGTGAAAAAATCCGTAACGTTTTCT CTTCTGTTAAAATGGCTAAAGAAAAAAAAGCTGCTGAAGCTGGTAACGCTACCGAAAACAAAGCTGAAtaa

\section{Idh}

ATGAAAAAAGTTAACCGTATCGCTGTTGTTGGTACCGGTGCTGTTGGTACCTCTTACTGCTACGCTATGATCA ACCAGGGTGTTGCTGAAGAACTGGTTCTGATCGACATCAACGAAGCTAAAGCTGAAGGTGAAGCTATGGAC CTGAACCACGGTCTGCCGTTCGCTCCGACCCCAACTCGTGTCTGGAAAGGAGACTACTCTGACTGCGGTACC GCTGACCTGGTTGTTATCACCGCTGGTTCTCCGCAGAAACCGGGTGAAACCCGTCTGGACCTGGTTGCTAA AAACGCTAAAATCTTCAAAGGTATGATCAAATCTATCATGGACTCTGGTTTCAACGGTATCTTCCTGGTTGCTT CTAACCCGGTTGACATCCTGACCTACGTTACCTGGAAAGAATCTGGTCTGCCGAAAGAACACGTTATCGGTT CTGGTACCGTTCTGGACTCTGCTCGTCTGCGTAACTCTCTGTCTGCTCACTTCGGTATCGACCCGCGTAACGT 
TCACGCTGCTATCATCGGTGAACACGGTGACACCGAACTGCCGGTTTGGTCTCACACCACCATCGGTTACGA CACCATCGAATCTTACCTGCAGAAAGGTACCATCGACCAGAAAACCCTGGACGACATCTTCGTTAACACCCG TGACGCTGCTTACCACATCATCGAACGTAAAGGTGCTACCTTCTACGGTATCGGTATGTCTCTGACCCGTATCA CCCGTGCTATCCTGAACAACGAAAACTCTGTTCTGACCGTTTCTGCTTTCCTGGAAGGTCAGTACGGTAACTC TGACGTTTACATCGGTGTTCCGGCTGTTATCAACCGTCAGGGTGTTCGTGAAGTTGTTGAAATCGAACTGAA CGACAAAGAACAGGAACAGTTCTCTCACTCTGTTAAAGTTCTGAAAGAAACCATGGCTCCGGTTCTGTAA

7. $f d$

$f d 1$

ATGGCACTCCGCACCATGGTTGACCCAGATACATGTACCTCATGCGAGCTGTGTTACGATCGCGTTCCGGAA GTATACAAAAACCGCGGTGATGGTATTGCTGAGGTGGTAAGTCCGGGACCGGATGGATGGATGATGGTACC ACCAGAGCTGGAGCAAGAGGTTAAGGAAGTTACCGACGAGTGTCCGAGTGGCTCTATCATTACAGAGGAA GTATGA

\section{$f d 2$}

ATGCGCATTCTCATTGACATTGATACATGCACAACCTGCCGCCTGTGCTACGATACGCTTCCTACTGTATTTGT GGACCGCGGTGATGGCATTCCGATTACACTGCCGATGAAAAGCTTCCCTGACCGCAACCTTGTAGAAGCTAT TAAAGAGGTGATGGAGAGCTGCCCGAGCAATTCTATTCAGATGGAAGAAGTTGGATAA

$f d 5$

ACCGAAGCTAAGATTCGTGTATGGATCGATCGCGAGCAGTGCATTGCAGACATGGTATGTGTAAGCCTCTGT CCTGATGTCTTCGAAATGAGTGAGGAGGATGGCAAGGCACAGATTGCTGCTAAGTGGCGTGTAGACAGTAA TAACCCTGCCGAGGGCCTCGTGCCGGCTGAACTCAAGGACTGTGTATCCGCAGCTGTCGAAGCCTGCCCTG TAAGCATCATTCACTTCGAGGAGAAGTAA

$f d 6$

ATGGGTCTGAAAGTTCGTGTTGACCAGGACACCTGCACCGCTTGCGAACTGTGCTACGACCGTATACCAGA 
AGTGTTCAAAAACGCCGGTGACGGTATCGCTGACGTTGTTAAATGCGACATCGAAGACGACGAAGGTTGCT GGATGATCGTTCCGGAAGGTCTGGAAGAAGAAGTTCAGGAAGTTGCTGACGAATGCCCGTCTGGTTCTATC ATCGTTGAAGAAGAATAA

$f d 7$

ATGGCTAAACTGAAAACCATGGTTGACCAGGAAACCTGCACCGCTTGCGAACTGTGCTACGACCGTGTTCC AGAAGTCTACAAAAACAGGGGTGACGGTATCGCTGACGTTGTTAAATGCGACATCAAAGACGAAGAAGAC CACTGCTGGATGATCGTTCCGGAAGGTCTGGAAGACGAAGTTCGTGAAGTTGAAGAAGAATGCCCGTCTG GTTCTATCATCGTTGAAGAACTGGAAGAATAA

$f d 8$

ATGAAATCTCTGGCTGAACTGGAAGAAATCCGTAAACGTACCCTGGACCAGATCAACCTGCGTACCTCTAAC CACGACATCCGTGTTGTTGTTGGTATGGCTACCTGCGGTATCGCTGCTGGTGCTCGTCCGGTTATGAACGCTT TCGTTGAAGAACTGAACAAACGTAACCTGACCAACGTTGCTGTTACCATGACCGGTTGCATCGGTATGTGCA AACTGGAACCGATCGTTGAAGTTATCGACAAAGACGGTTCTAAAGTTACCTACGTTAAAATGACCCCGGAAA AAGTTGCTCGTGTTGTTGCTGAACACATCGTTAACGGTCGTGTTTGCACCGAATACACCATCGCTGAAGCTG ACAAATAA

$f d 9$

ATGAACAACTTCACCAAAATCCAGGACAAACTGATGGAAATGGGTGCTTCTTTCGTTGGTTGCTCTGACGTT GAAGCTTACCTGCCGGAAAACCTGAAAAAAGTTAAATACGCTATCACCGTTGGTGTTCGTCTGTCTGACTTC ATCATCGACCAGATCTCTGACAAACCGACCTACACCTACTTCCACCACTACCGTGCTGTTAACACCCTGATCG ACCAGATCACCCTGCGTGGTCAGATCGAAATCCAGAACATGGGTTACTCTGCTATGGCTGTTCCGGCTTCTCA GACCGTTAACGACCTGGAAGACCGTTACTCTGGTGTTTTCCAGCACAAAACCGCTGCTGTTAAAGCTGGTCT GGGTTGGATCGGTAAAAACGGTCTGTTCATCTCTACCAAATACGGTCCGCGTGTTCGTCTGGGTACCATCCTG ACCGACATGGAACTGCCGATCGGTTCTCACGTTGTTCGTGACGGTTGCGGTTCTTGCCGTATCTGCGTTGAC TCTTGCCCGGCTATGGCTCTGACCGGTAACTCTTGGTTCGCTGGTTGCGACCGTTCTCACATCGTTGACGCTC 
AGGCTTGCTCTGAATACATGAACCGTAACTTCAAACACATCGGTCGTGGTTCTGTTTGCGGTATCTGCATCCG TGTTTGCCCGAAAGGTTTCTCTAAATAA 